\title{
UN ATELIER DE MOSAÏQUES TARDIVES EN PROVENCE
}

\author{
par Henri LAVAGNE
}

L'évolution de l'art de la mosaïque en Provence est relativement bicn connue pour sa période ancienne. La majeure partie des séries analysées ${ }^{1}$ est constituée de pavements géométriques dont les canevas sont hérités directement de modèles italiques du $\mathrm{I}^{\mathrm{er}}$ siècle, caractérisés par l'emploi du noir et blanc, et dont le style n'atteint à une véritable originalité qu'à partir de la seconde moitié du II $^{\mathrm{e}}$ siècle, lorsque la polychromie et la variété des remplissages ornementaux commencent à s'imposer. Cette vue d'ensemble n'a II ien de surprenant dans la partie la plus romanisée de la provincia, où des villes comme Vìmes, Glanum, Orange, Avignon, attestent par leur parure monumentale, la rapidité avec laquelle les courants artistiques venus de Rome et de l'Italie du nord, se sont jépandus ${ }^{2}$. En revanche, dans le tableau généralement admis que l'on donne de la Narbonnaise à partir de la fin du III ${ }^{\mathrm{e}}$ siècle, il apparait que la mosaïque n'occupe aucune place. On insiste sur le climat d'insécurité créé par les invasions barbares et les dissensions intérieures, on met en valeur le ralentissement des échanges, on souligne l'abondance des traces d'incendie, et on dresse le sombre bilan des habitats désolés et des cités qui se resserrent à l'étroit derrière des remparts édifiés à la hâte avec les débris d'époques plus fastes. Dans cette vision trop schématique, les arts du décor, et particulièrement la mosaïque, semblent avoir disparu ${ }^{3}$. Or, des documents jusqu'ici méconnus montrent l'existence d'au moins un atelier qui produit des œuvres de grande qualité à la fin du Ive ou au début du ve siècle.

Un premier exemple nous est fourni par un groupe de mosaïques de Saint-PaulTrois-Châteaux (Drôme) mises au jour au xix ${ }^{\mathrm{e}}$ siècle, mais qui, pour des raisons diverses,

1 Sur ces traits principaux, cf. notre fascicule du Recueil général des mosaiques de la Gaule, III, .Varbonnaise, I, Confédéralion Catare, $\mathrm{X}^{\mathrm{e}}$ suppl. à Gallia 'sous presse:. Le deuxième fascicule consacré aux mosaĭques trouvées dans les territoires des peuples de la Confédération des Voconces est en préparation.

2 Sur cet aspect, cf. en dernier lieu les actes du Colloque sur la Gallia romana, Rome, 1971, Acc. Naz. Lincei, 1973, quaderno no 158, nolamment p. 167-168.

3 Dans un domaine voisin, la sculplure, on est étonné de voir la paureté des lémoignages de "l'ècole" marseillaise ", malgré les efforts de F. Benoit pour melt re en lumière son essor au Bas-Empire. Cf. F. Brxort, Sculptures d'arl provincial au Bas-Empire en Provence, dans Provence hislorique, I, 1950-1951, p. 16-31. 
ont échappé à l'attention des spécialistes ${ }^{4}$. Ces mosaïques étaient déjà probablement connues des érudits locaux qui, dès 1710 , signalent " "plusieurs grands pavés à la mosaïque " au quartier Saint-Jean, dans les caves d'habitations bâties contre le rempart du BasEmpire ${ }^{6}$. L'archéologue avignonnais, A. Sagnier, qui assista à l'extraction de la plus grande partie des fragments en $1889^{7}$, indique qu'ils étaient situés "dans la cave de la maison de M. V..." sans autre précision. Le Musée Calvet d'Avignon ayant refusé d'en faire l'acquisition, le propriétaire fit déposer une cinquantaine de morceaux, représentant une surface "d'environ $30 \mathrm{~m}^{2}$ ». Dès lors, on perd leur trace. G. Lafaye, dans son Inventaire des mosaïques de la Gaule, paru en $1909^{8}$, puis J. Sautel dans le fascicule de la Forma Orbis Romani $^{9}$ consacré à la Drôme, ne font que reprendre la bibliographie ancienne sans localiser l'endroit où étaient déposés ces fragments, et sans les décrire. Or, d'un autre côté, nos recherches pour le Recueil général des mosaïques de la Gaule nous avaient conduit à analyser une séric de dix éléments de mosaïques de même style, utilisés pour paver le rezde-chaussée de l'hôtel particulier de l'érudit et collectionneur Paul de Faucher (1840-1907) à Bollène (Vaucluse). Cies mosaïques étaient données comme provenant du hameau de Chabrières, situé à quelques kilomètres de Bollène. En voulant préciser cette tradition orale, nous avons découvert qu'il s'agissait, en réalité, des derniers restes des mosaïques trouvées à Saint-Paul-Trois-Châteaux en 1889. En effet, un tirage à part de l'article d'A. Sagnier relatant les fouilles faites au moment de la dépose des mosaïques, avait été offert à $\mathrm{P}$. de Faucher, et celui-ci avait indiqué de sa propre main qu'il avait récupéré une partie de ces mosaïques pour en orner le rez-de-chaussée de sa demeure à Bollène. En outre, il ajoutait le nom du propriétaire de la cave en question, un certain M. Vallos. Grâce à ce nom. il était aisé de situer exactement l'emplacement des pavements en consultant l'ancien cadastre de 188:10 : l'angle sud-ouest de l'enceinte du Bas-Empire, entre l'actuelle rue Saint-Jean et le Cours Haut (fig. 1).

Cette zone est particulièrement riche en témoignages archéologiques qu'il importe de rappeler, car ils constituent les éléments d'une chronologie relative qui permet de mieux cerner la place des mosaïques. En 1801, deux bas-reliefs avaient été découverts

1 C̈est grace a l’aide ceflicace du br Henri de lanucher, qui a bien voulu faire de longues recherches dans les archives de son oncle, que nous avons pu identifier ces pavements. Nous tenons également a remercier M. P. Pradal, Président du Syndicat d’Iniliative de Bollene, II. (.l. Boisse et les membres de la Société d'archéologie de Saint-PaulTrois-chateaux dont l'accueil cordiat ot empressé nous a été d'un grand secours durant cette enquêle.

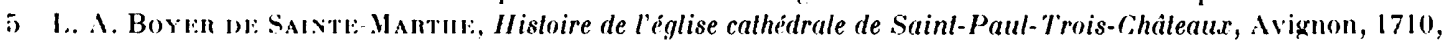
p. 4.

6 X. Delachorx, Essai sur la slalistique el les anliquilís du déparlement de la I)rime, Valence, I817, p. 309;

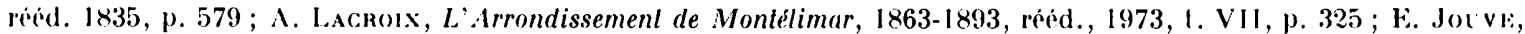
slatislique monumentale du département de la Irôme, valence, 1867, p. 357.

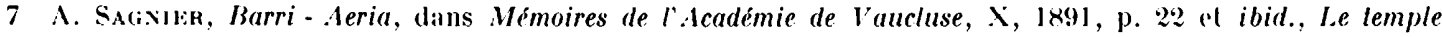
d.Augusle à Saint-Paul-Trois-Chateaur, VIII, 1889, p. 963-975. Repris par 1.. FIL.6r, Histoire de Saint-Paul-TroisChâteaur, dans Bulletin de la Sociple d'archóologie el de slalistique du díparlement de la Drime, $1910,0.220$.

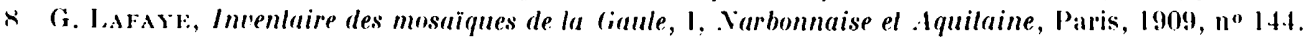

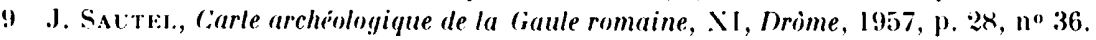

10 Maison Vallos ou Vallosse. n* 942 de la matrice cadastrale; section (), "Boure "; parcelles 546, 547, 548 maison el jardin : 


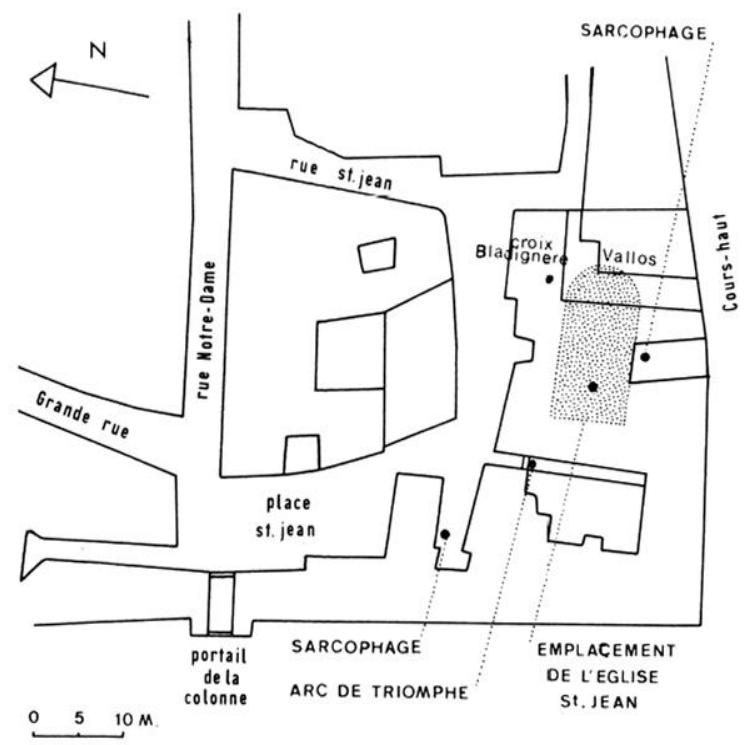

l Saint-Paul-Trois-Chàteaux. Siluation de l'église Saint-Jean.

dans la maison contiguë à celle de M. Vallos"11. N. Delacroix, en $1817^{12}$, décrit " une portion de murailles qui porte des colonnes d'un goût exquis " et A. Sagnier, en 1889, "les restes d'un portique construit en gros blocs de pierre sans ciment, dont la baie présente une hauteur de $3,50 \mathrm{~m}$... et une largeur de $1,45 \mathrm{~m}$ à son plus grand diamètre ", au-dessus de laquelle "existe une large niche bordée de divers trous qui semblent avoir servi à des crochets pour assujétir une plaque de marbre ou de bronze ". A côté, il note "une autre niche encore plus grande" dont il ne s'explique pas la fonction; enfin, trois pilastres cannelés $(2,50 \mathrm{~m} \times 0,40 \mathrm{~m})$ utilisés en réemploi, et dans la cave voisine « d'énormes quartiers de pierre qui paraissent avoir servi de fondations ». Aujourd'hui, un pilier cannelé de retombée d'arc est encore visible ainsi qu'un cintre et une partie de parement en grand appareil. Tout ceci confirme l'hypothèse d'un arc de triomphe ${ }^{13}$ qui pourrait avoir eu comme métopes les bas-reliefs trouvés au début du xıxe siècle à proximité immédiate. L'importance de ces témoignages architecturaux est encore accrue par la présence de très nombreux débris de sculptures utilisés comme assises pour le lit de pose des mosaïques. A. Sagnier nous en donne une liste détaillée : "grands rinceaux de feuilles d'acanthes de haut style corinthien ou composite ; au-dessous de ces feuilles se déroulent deux longs filets ou chapelets d'oves, de grandes perles ou autres objets, et l'intervalle des deux filets est occupé par une large

11 E. (:HAplír, Leltre à .Y. Le Inirecleur de la Revue du Dauphiné et du Vivarais, dans Revue du Dauphiné et du Vivarais, 2, 1878, p. 249-254. Sur l'un, on voyait "Jupiter et une nymphe allaitant "; sur l'autre, un *amour sur un char trainé par deux lions ". I)essin conservé dans le fonds Gariel à la Bibliothèque de Grenoble. En réalité, le premier, conservé aujourd'hui dans une collection privée à Saint-Paul-Trois-Châteaux, représente Léda et le cygne. Il est intéressant de noter que le sujet est également figuré sur l'are de triomphe de Reims, traditionnellement appelé "Porte de Mars " cf. G. (Ch. Picarl), La Porte de Mars a Reims, dans Acles du 9.je Congrès nalional des Sociélés savantes, Reims, 1970, Paris, 1974, p. 59 ).

12 .. Detackolx, op. cil.,p. 309

13 Hypothese due à (il. Bolsse, Le quartier scint-Jean, dans Tricasini, 1, 1974, p. 32. Sur ce type d'ares, cf. (i. A. Maxst:Lt.1, Aedicula columnis adornala. Nuove osservazioni suyli archi romani italici e provenzali, dans Mélanges 1. Benoil, N Inst. Inler. Éludes L.igures , 1972, p. 103-109. 
bande de lignes géométriques entrecroisées. Le tout se termine en haut et en bas par de larges moulures plates... » et d'autre part "des feuilles d'acanthes détachées qui paraissent avoir servi de modillons ou d'antéfixes ". La présence d'un édifice très probablement du Haut-Empire dans ce secteur est donc certaine. Nous ne suivrons pas Sagnier lorsqu'il tire argument de trois inscriptions mentionnant des sévirs augustaux à Saint-Paul pour conclure qu'à cet emplacement s'élevait le temple de Rome et d'Auguste ${ }^{14}$. L'hypothèse serait trop hasardeuse et n'intéresse d'ailleurs que la période précédant l'exécution des mosaïques. Il suffit de retenir que celles-ci ont été établies sur les restes d'un bâtiment public, civil ou religieux important, à en juger par sa décoration sculptée.

Des $30 \mathrm{~m}^{2}$ de pavement dégagés en 1889, il ne subsiste que dix fragments de dimensions très inégales sur les cinquante qui avaient été déposés. Huit d'entre eux ont subi d'importantes restaurations au moment où ils furent installés dans un sol moderne, en ciment rougeâtre, parsemé de cubes noirs, qui sert de fond à l'ensemble. Les deux derniers (tapis E, voir ci-dessous, et fragment du tapis D) n'ont pas été réinsérés dans le pavement du vestibule de l'hôtel de Faucher, ce qui permet de voir que les cubes reposent sur une couche de béton fin, piquetée de minuscules éclats de tuileaux (épaisseur $2 \mathrm{~cm}$ ) extrêmement dure, elle-même placée sur un mortier de pierrailles noyées dans un ciment plus friable (10 cm d'épaisseur). Les tesselles sont régulièrement posées et ont une surface un peu inférieure à $1 \mathrm{~cm}^{2}$ en moyenne.

Le tapis le plus important est double $(4,26 \mathrm{~m} \times 2,31 \mathrm{~m})$. Il est entouré d'une bande blanche (cinq rangs de cubes, trìs restaurés) el d'une tresse polychrome (largeur $10 \mathrm{~cm}$ ), dont les teintes dominantes sont une couleur heige tirant sur le bistre, un blanc très clair (calcaire) alternant avec un rouge (Lerre cuite), un bleu (marbre translucide), un jaune, cernés d'un filet noir. Le tapis $A$ (fig. 2) est formé par un rectangle de $2.13 \mathrm{~m} \times 1,33 \mathrm{~m}$ oì s'inscrit un cercle (diamètre $1,22 \mathrm{~m}$ ). langent à l'un des petits còtés et laissant à l'autre extrémité une partie plus large, prolongée par une bande de raccord horizontale (largeur $12 \mathrm{~cm}$ ) qui comporte une rangée de carrés à degrés polychromes se touchant par les pointes, les triangles résiduels étant également à degrés, mais blancs. De ce còté, les écoinçons sont garnis de trois bulbes maladroitement restaurés, entourés de deux feuilles d'hederae. Les deux autres écoingons, ègalement modernes, sont moins malhabiles, et les bulbes qui les décorent sont ornés d'un motif de chevrons polychromes en arc-en-ciel. Les mèmes tiges terminées en hederae, dont l'ap $\varepsilon x$ est très effilé, les entourent. Le cercle - à part des réfections limitées à la bande blanche - est de facture antique. Il est garni d'une bordure intérieure $(11 \mathrm{~cm})$ en ruban ondé dont chaque élément est alternativement bleu/histre/blane et rouge/bistre/ blanc. Les deux filets noirs qui entourent cette bordure sont agrémentés d'une ligne pointillée de cubes noirs. Aprìs une nouvelle bande blanche (3 rangs de cubes), cernée d'une ligne de tesselles marron clair, le cercle intérieur (diamètre $78 \mathrm{~cm}$ ) est chargé d'une étoile de huit losanges dessinée par des filels noirs. Les losanges ont un remplissage polyclirome qui alterne : brun rouge/bistre/ blanc et bleu/bistre/blanc. Au centre de chacun, un petit losange emboîté noir. Dans les triangles résiduels déterminés par les branches des étoiles, motifs triangulaires en arc-en-ciel avec le même jeu de couleurs alternées que dans les losanges, les dégradés étant soulignés par des lignes de cubes noirs en chevrons.

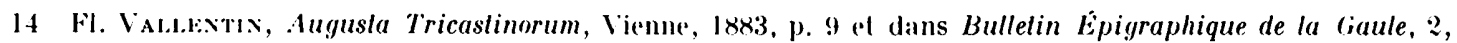
1882, p. 227, $n^{\circ}$ 1. L'inscription CIL XII, 1731, est conservée dans lat collection P'. de Faucher à Bollène. L'inscription

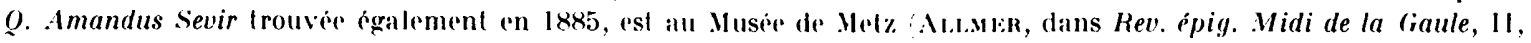
p. $131, n^{\circ} 553$. 


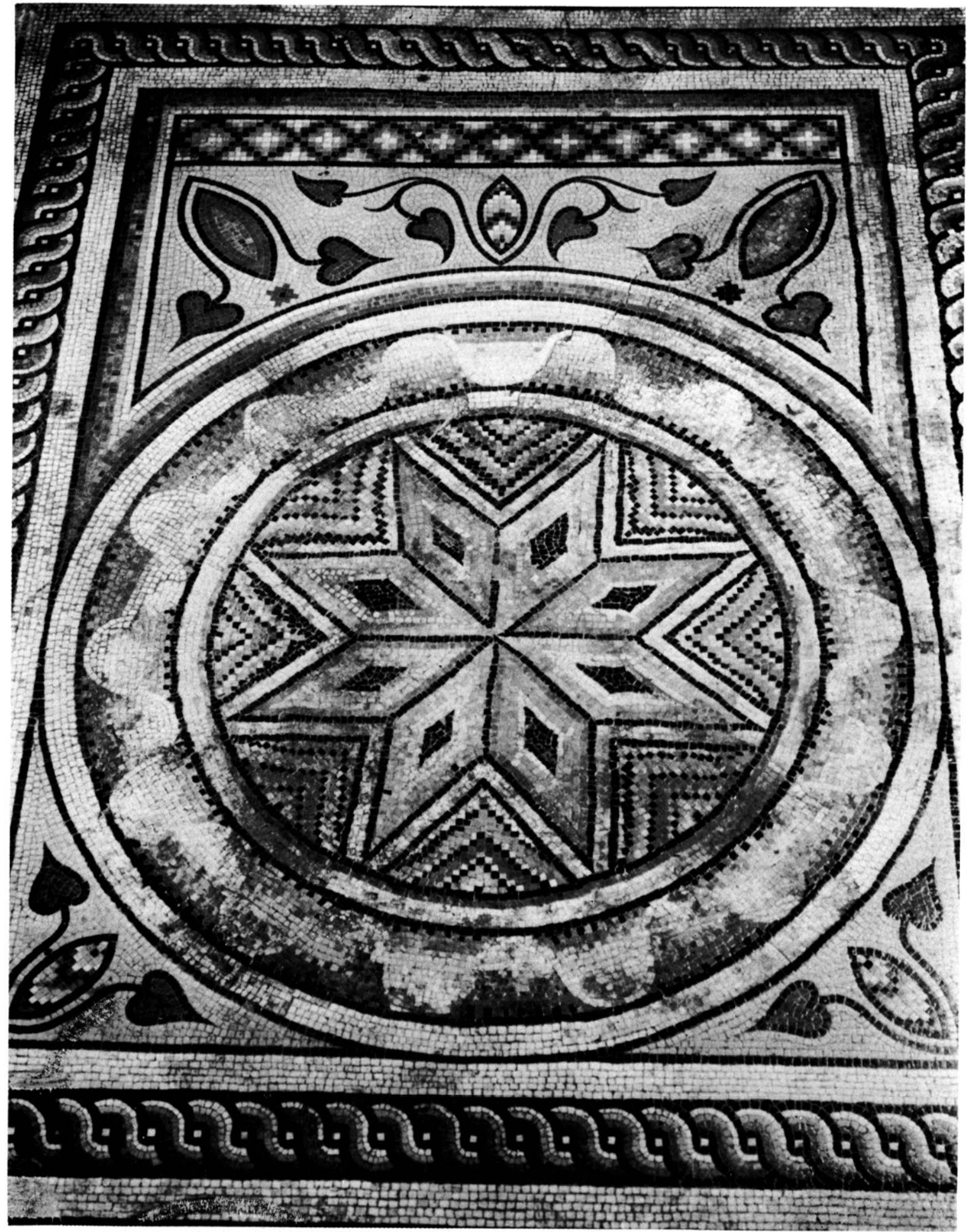

2 Mosaïque $A$ collection particulière à Bollentr, Vaucluse 
Le tapis 13 (fig. :3) relié au précédent par la tresse (:2,1:3 m $\times 0.98 \mathrm{~m}$ ), offre une composition de huit quadrilobes de peltes entourant un nœud central en sparterie double. Les peltes attestent également des restaurations, mais plus soignées que dans le précédent fragment. Dans chaque motif (largeur $43 \mathrm{~cm}$ ), les brins de la sparterie cernés de noir reproduisent les irisations de couleurs déjà notées : deux rangs rouges, un rang blanc, deux de couleur mastic, un rang blanc. Les peltes sont blanches, cernées d'un filet noir, et elles sont ajourées au centre par un motif évasé noir, en forme d'accent circonflexe. Les triangles curvilignes du fond (déterminés par les lignes courbes des peltes), sont bleus.

Le tapis C est composé de trois fragments disjoints enchassés dans le sol en ciment moderne sans qu'il ait été possible de les regrouper en un ensemble cohérent. Le plus grand fragment $(1,56 \mathrm{~m} \times 0,80 \mathrm{~m})$, entouré de la tresse polychrome déjà décrite pour les tapis $\mathrm{A}$ et $\mathrm{B}$, est une composition en imbrication d'écailles (fig. 4). Sept rangs d'écailles se succèdent et leurs couleurs sont variables sans recherche de symétries : les unes sont entièrement blanches, les autres n'ont qu'un filet double de cette couleur, le cœur de l'écaille elle-même étant tantòt orange, tantôt brun clair, voire jaune. Toutes ont en commun un motif en fer de lance noir, qui dessine comme un dard placé au point de tangence de chaque écaille avec la suivante. Le second fragment d'écailles $(0,90 \mathrm{~m} \times 0,55 \mathrm{~m})$ ajoute, en outre, une bordure en damier polychrome. Six carrés successivement rouge, jaune, blanc, bleu, rouge, jaune en constituent les cases. Un troisième fragment d'écailles (fig. 5) $(0,85 \mathrm{~m} \times 0,57 \mathrm{~m})$ est lié à l'amorce d'un motif de cercle traité en ruban ondé comme dans le tapis A. Mais à l'intérieur du cercle, on note le début d'un câble en arc-en-ciel (largeur $12 \mathrm{~cm}$ ) où les cubes sur la pointe reprennent le jeu des couleurs déjà relevées, blanc/noir/rouge, blanc/bistre/ noir, bleu/blanc/rouge et noir/blanc/bistre.

Le tapis I) est fragmenté en trois éléments qu'il est impossible de réunir pour former un tout. Le morceau essentiel $(0,82 \mathrm{~m} \times 0,84 \mathrm{~m})$ est l'angle d'une bordure en natte (fig. 6$) \mathrm{de} 33 \mathrm{~cm}$ de large. A l'une des extrémités s'amorcent des solides polychromes vus en perspective, sur fond noir. Dans l'angle inférieur, un petit carré (fig. 7) $(18 \mathrm{~cm} \times 19 \mathrm{~cm})$, où le décor en arc-en-ciel en cubes posés en diagonale est particulièrement riche (15) couleurs différentes), n'est peut-ètre que l'angle d'un motif d'une surface vraisemblablement plus large. La natte a une variété de couleurs moins grande : brins blancs, bistres, bleus, succédant à des brins blancs, bistres, rougcs. Un deuxième fragment de la natte $(0,82 \mathrm{~m} \times 0,61 \mathrm{~m})$ pourrait avoir été son prolongement d'un còté. Le troisième morceau (fiğ. 8) est un élément du tapis de solides vus en perspective $(0,64 \mathrm{~m} \times 0,62)$ dont il a la même polychromie (bistre, rouge, bleu; extrémité carrée, blanche).

Un tapis E devait oflrir un décor d'entrelacs compliqués (fig. 9) où cercles petits et grands étaient traités tantòt en càbles en arc-en-ciel (rouge, blanc, bistre), tantòt en tresse polychrome (brins, bistre, blanc el bleu, bistre). Dans l'un des quarts de cercle visibles, une bordure à fond bistre est ornée de denticules noirs. Dans l'autre, on distingue un carré sur la pointe dont le côté est dessiné par une ligne de petits carrés formés de quatre tesselles adjacentes, se touchant par les angles.

Enfin. un fragment de bordure $(15 \mathrm{~cm} \times 10 \mathrm{~cm})$ est isolé : postes noires. très raides, avec un seul enroulement en forme de (; mạuscule.

Cette description ne donne qu'une faible idée de la richesse de la polychromie de ces fragments. La qualité technique du dessin (notamment dans l'entrecroisement des brins de la natte) est excellente et va de pair avec la finesse des différentes teintes. L'absence de relevé avant la mutilation de l'ensemble est aggravée encore par l'aspect disparate des décors. Ln point est sùr, il est impossible de placer dans une composition d'ensemble less éléments conservés. C'est la raison pour laquelle nous arons préféré les regrouper en tapis différents. Si l'on rappelle que nous n'avons que dix fragments sur les cinquante qui avaient été prélevés, on peut conclure que nous avions à l'origine une surface au sol divisée 


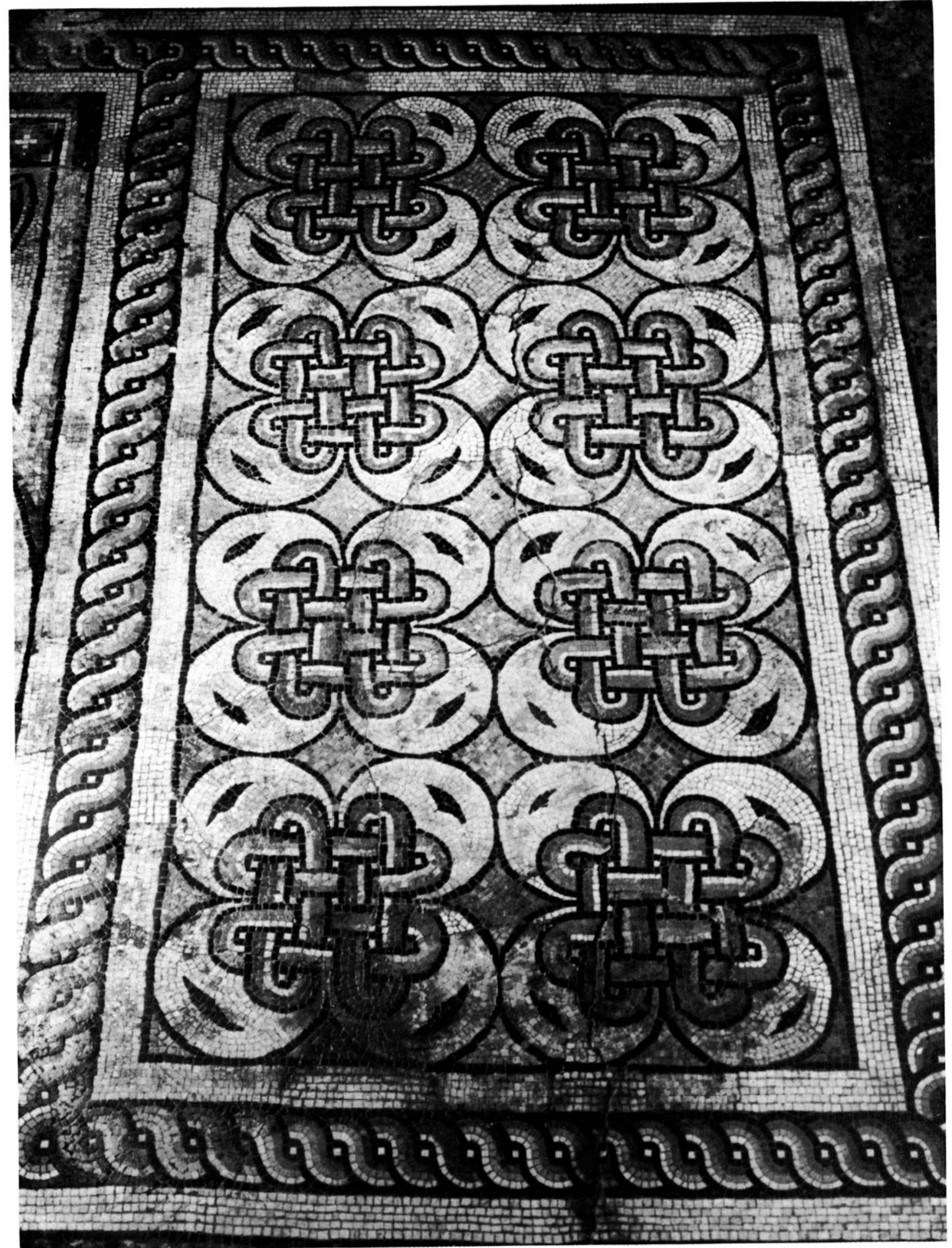

3 Nosatique B. 

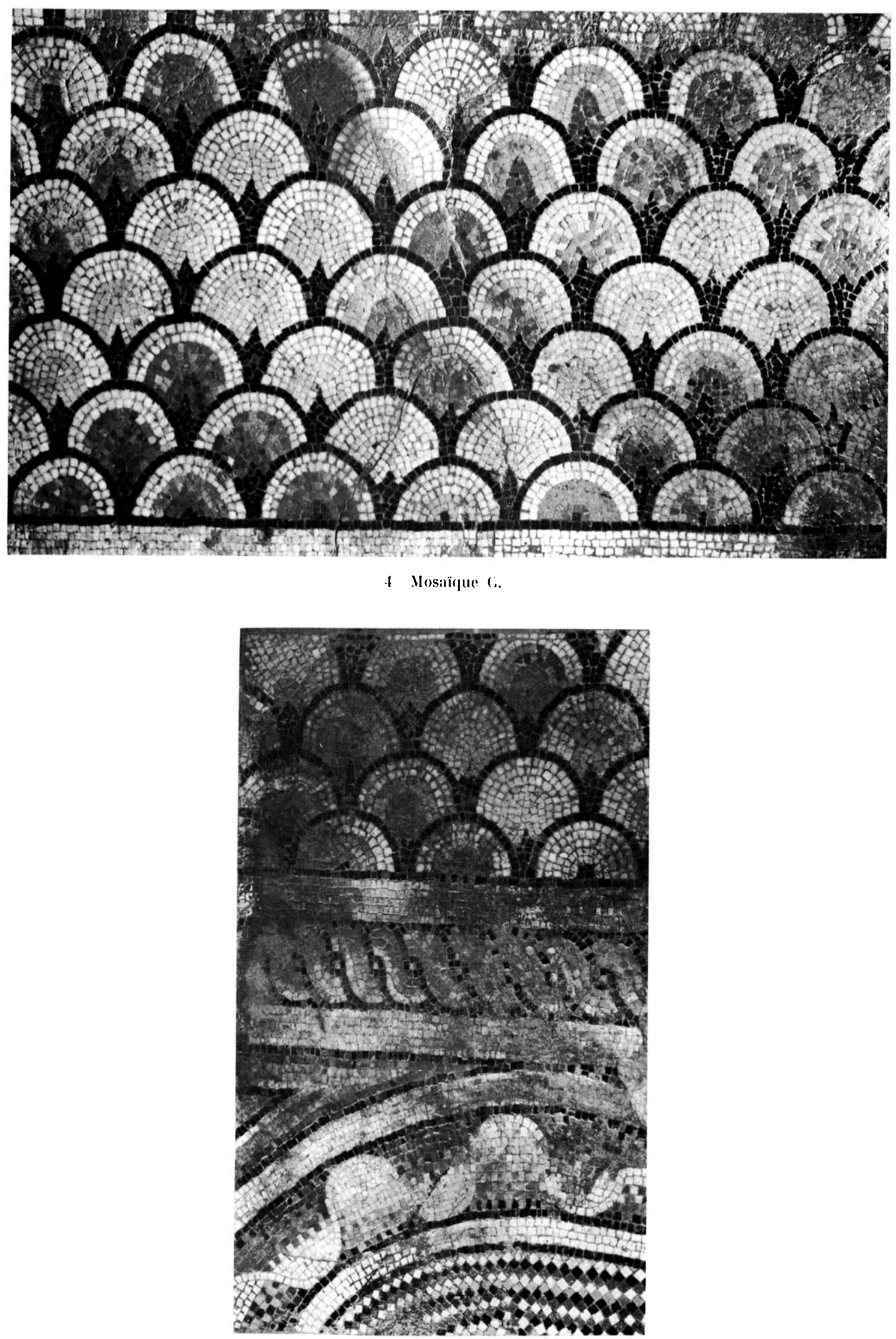

5 Elcment du talpis $(.$. 


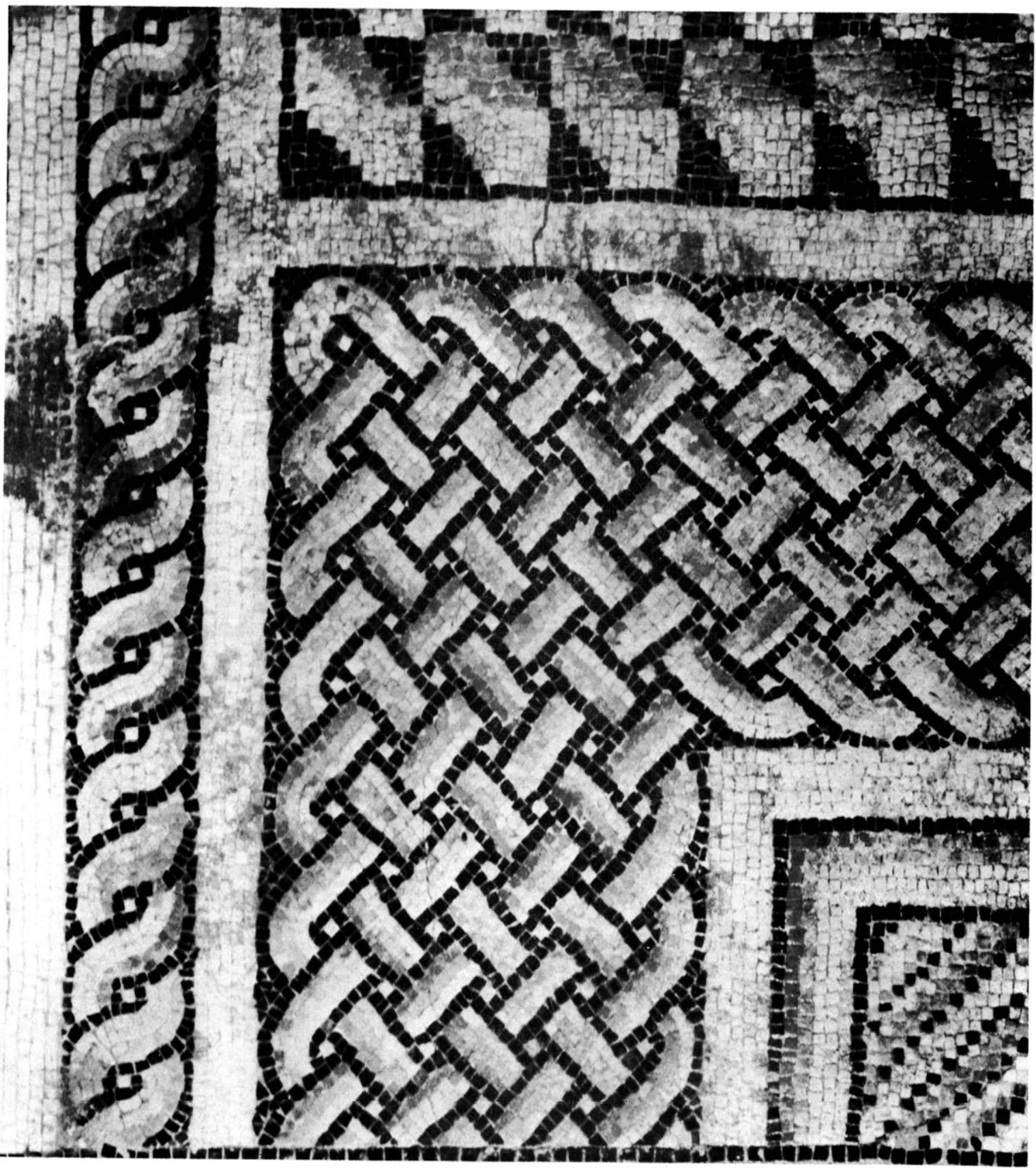

6 Mosaïque D. 


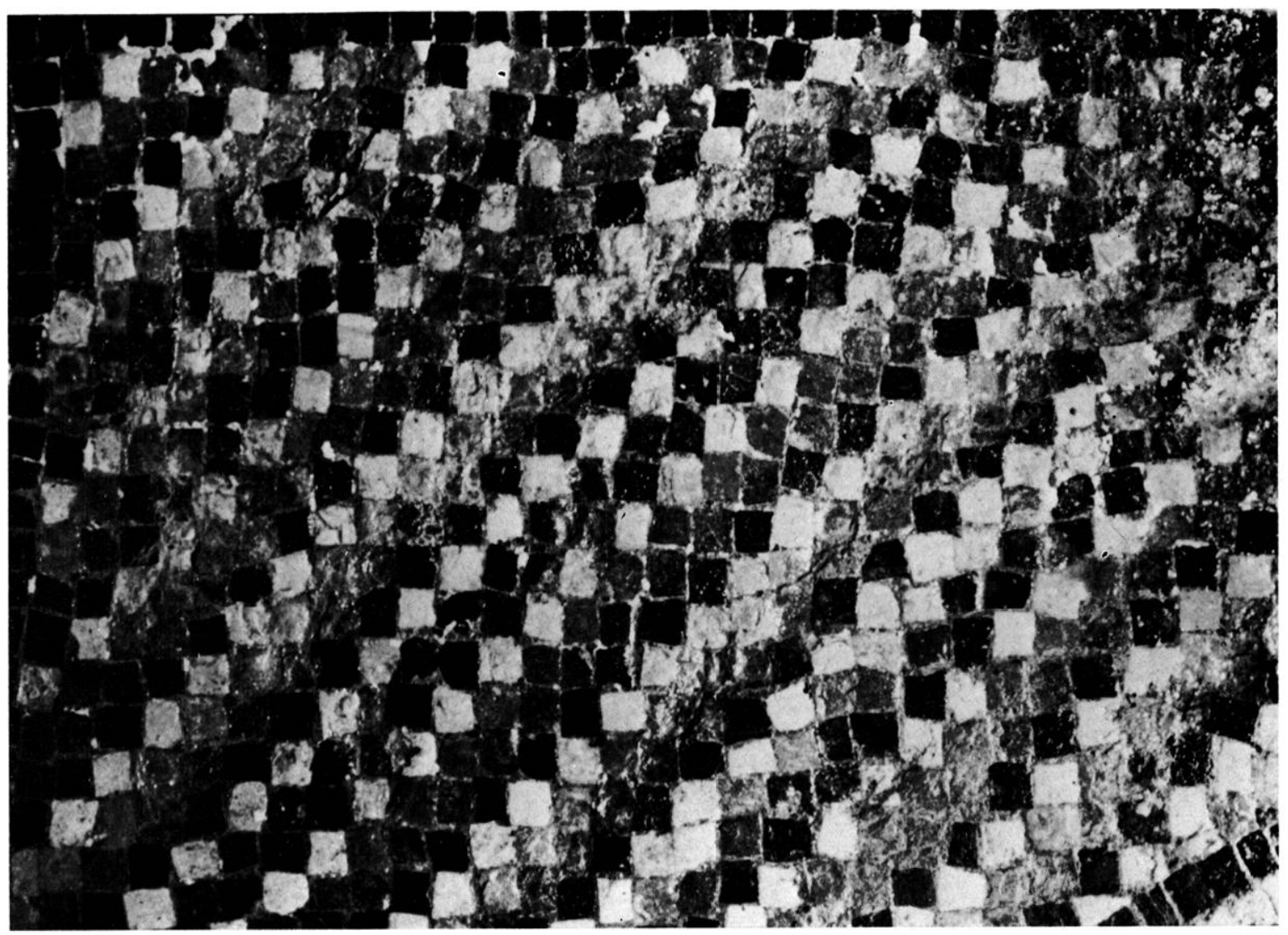

7 Élément du tapis I).

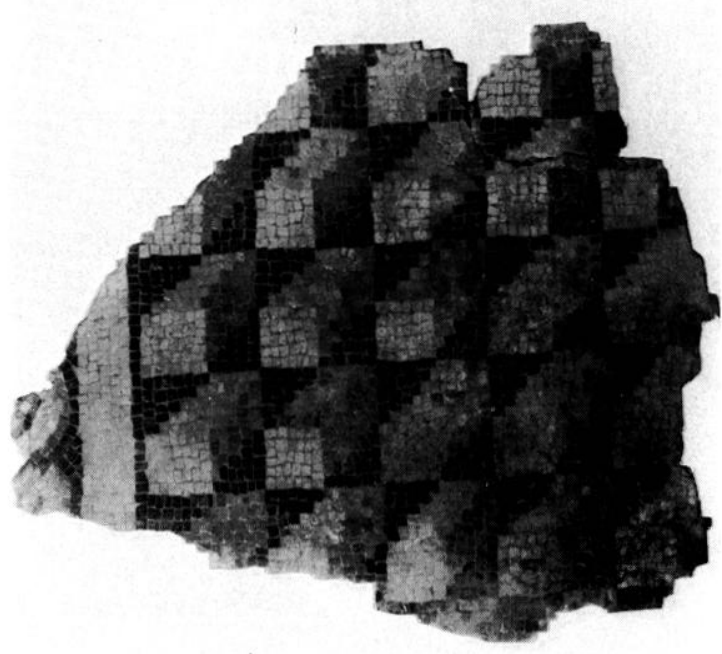

$x$ filement du lapis I)

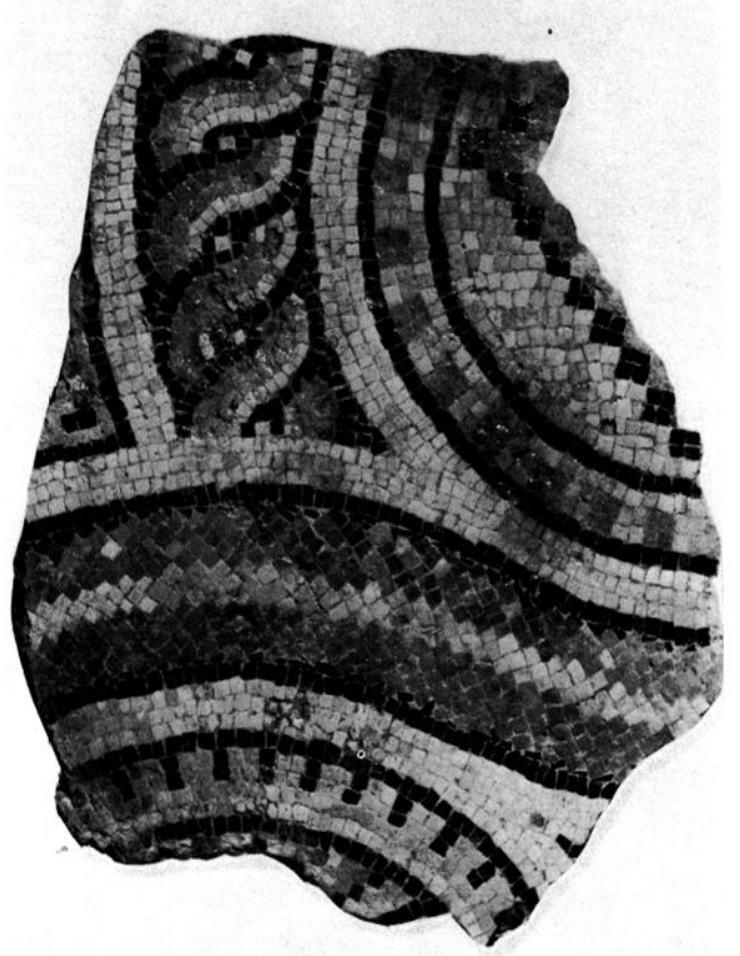


en tapis contigus, ce qui amène à imaginer une salle de grande étendue, dont l'espace était divisé en plusieurs zones. On peut penser, par exemple, à une salle à abside. à un plan basilical, etc.

La datation de ces mosaïques ne peut être fondée que sur des considérations stylistiques, aucun indice stratigraphique n'ayant été donné lors des fouilles de 1889. Il est clair que les canevas de composition, les motifs de remplissage et la palette des coloris employés, concordent pour faire attribuer d'emblée ces pavements au Bas-Empire. Est-il possible de préciser davantage?

Le décor le plus caractéristique est celui des quadrilobes de peltes (tapis I3, fig. 3). Le schéma qui allie les peltes à un motif central présente de nombreuses variantes. La plus commune est celle qui consiste à placer les quatre peltes sur les còtés d'un carré orné, le plus souvent, d'un noud de Salomon ${ }^{15}$, ou d'un motif animé (volatiles) comme dans le baptistère d'Oued-Ramel ${ }^{16}$, ou dans quelques exemples d'époque paléochrétienne en Italie du nord, notamment à Aquilée ${ }^{17}$. R. Farioli en signale un cas comparable dans l'église de la Sainte-Croix à Ravenne ${ }^{18}$ qu'elle date du ve siècle.

Plus proche géographiquement de Saint-Paul, et toujours en milieu chrétien, on citera les dessins représentant les mosaïques, aujourd'hui disparues, du baptistère de Marseille, qui sont également attribuées à la fin du $\mathrm{v}^{\mathrm{e}}$ ou au début du ve19. Dans tous ces exemples, les motifs sont centrés sur un quadrillage dessiné par un filet qui relie chacun des quadrilobes. A Saint-Paul-Trois-Châteaux, les peltes sont placées en position diagonale par rapport au milieu du motif et sont tangentes entre elles. Elles sont donc assez différentes de celles de l'Italie du nord ou d'Afrique. On ne peut les comparer qu'à des mosaïques de Grèce ou du Proche-Orient. A Thessalonique, Ph. Petsas a publié brièvement un pavement dont nous ne pensons pas qu'il puisse être antérieur à la fin du Ive siècle ${ }^{20}$ et qui présente exactement le même quadrilobe que le nôtre, mais il alterne avec un autre type dont le centre est non pas un nœud en sparterie double, mais un fleuron échancré de quatre-feuilles cordiformes. Même variante dans une villa tardive à Argos ${ }^{21}$. Les mosaïques de cette villa offrent également des bulbes décorés de chevrons polychromes, un noud d'entrelacs traité en câble et tresse, et des postes à enroulement très raides. Tous ces détails sont présents, comme on l'a vu plus haut, dans les parements de Saint-Paul-Trois-(hâteaux. Au Proche-(Orient, on repère ce motif à trois reprises : dans le marlyprium de Misis ${ }^{22}$, que

15 Ln exemple parmi tant d'autres au Musée de Tripoli, dans S. Alralamma, L'Italia in Africa, I, I, I Mosaici, Rome, 1960, pl. 47. Nous soulignons cet exemple qui se rapproche des nòtres par le noud en sparterie au centre du carré. P1. 61, on trouvera une variante ou les peltes sont disposées autour d'un carré sur la pointe.

16 H. SterN, Le décor des pavements et des cuves dans les baptislères paléochróliens, dans Acles du I’e Congrís inlernational d'Archéologie chrétienne, Aix-en-Provence, 19.5t, Paris, 1957, p. 382, fig. 1.

17 A Aquilée, dans G. Brusix, P. I. Zovatto, Monumenti paleocristiani di Aquileia e di (irado, I'dine', 1957 , p. 358-361, fig. 142-143. Exemple daté du ve siècle.

18 R. Farioli, Pavimenti musivi di Ravenna paleocristiana, Ravenne, 1975, p. 85, fig. 31.

19 X. Barral. Y Altet, Le baptistère de Marseille, dans Archeologia, 1974, no 73 , fragment $\mathrm{F}$, p. 8 et $1 \times$.

20 Ph. Petsas, The agora of Thessalonili, dans Arch. Anal., 1968, p. lō, fig. 2.

21 Fouilles J.-P. Sontxi, dans Bull. corres. hellénique, 92, 1968, p. 1041, figr. 4. Celui-ci nous a confirmé que la dale la plus probahle en altendant létude des monnaies trouvées sous le parement lui paraissait être la deuxieme: moitié du rve siecle.

22 L. BudDE, Antike .Mosaiken in Kilikien, I, 1969, pl. 49, 65. 
nous datons avec E. Kitzinger ${ }^{23}$ du ve siècle, à Gerasa, au dernier quart du rve siècle selon D. Levi ${ }^{24}$, et à Antioche même, dans la maison de la déesse marine $e^{25}$ daté de la deuxième moitié du ve siècle, mais déjà plus stylisé que les précédents (le fond est ajouré autour de noud de Salomon). Le seul exemple qui soit approchant en Occident se trouve à Aquincum en Pannonie ${ }^{26}$, où la date proposée est très antérieure à la série que nous avons relevée (début du ${ }_{11}{ }^{\mathrm{e}}$ siècle), mais il faut noter que l'effet est très différent, puisque les peltes sont placées sur les brins latéraux du nocud en sparterie, et non pas en angle, et que l'ensemble est enveloppé dans une roue de peltes qui circonserit le quadrilobe.

Les autres motifs sont moins caractéristiques et appartiennent à un répertoire beaucoup plus répandu. Toutefois, le noeud de sparterie est, lui aussi, un des poncifs traditionnels dans les mosaïques du Proche-Orient ${ }^{27}$. beaucoup plus qu'en Occident, de même que les carrés remplis par des lignes de cubes en diagonale que nous avons dans le tapis $\mathrm{D}^{28}$. Enfin, nous reconnaissons aussi les trois modes de traitement favoris dans les pavements orientaux : la tresse, le câble en arc-en-ciel et le ruban ondé, dont les jeux de couleurs créent des effets d'irisations translucides. Dans les imbrications d'écailles, on remarque le détail des pointes noires en fer de lance bien attesté dans de nombreux exemples à Timgad ${ }^{29}$. mais également dans des pavements tarlifs de Gaule, à Blanzy-les-Fismes ${ }^{30}$ et Montcaret (I)ordogne) ${ }^{31}$.

Ce relevé rapide des parallèles et des motifs identiques nous permet donc de placer l'exécution de ces mosaïques à la fin du $\boldsymbol{w}^{\mathrm{e}}$ ou au début du ve siècle. La rareté du décor du tapis de quadrilobes implique d'ailleurs une circulation de "carnets de motifs " qui participe de la grande "Koinè " des répertoires du Bas-Empire. Le seul ensemble de mosaïques galloromaines qui présentent un style analogue à celui des fragments de Saint-Paul-TroisChâteaux est le groupe de Loupian (Hérault) ${ }^{32}$ que nous avons proposé de dater également du début du ve siècle, mais le fait est plus frappant dans la vallée du Rhône que sur la còte méditerranéenne où les échanges par les ports en liaison avec l'Orient font comprendre plus facilement les affinités de décor. D'autre part, dans le cas de Loupian, il s'agit d'une de ces nombreuses villas tardives de riches propriétaires fonciers qu'on commence aujourd'hui à mieux connaître par les exemples de l'Aquitaine. Mais en milieu urbain, à Saint-Paul-

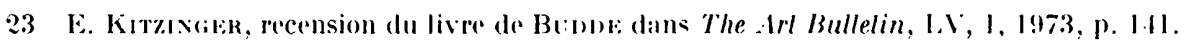

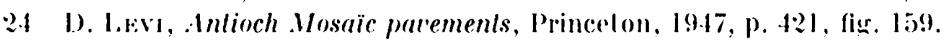

25) Ibid., pl. (:X.XII, c.

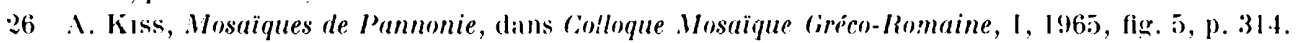

27 On cilern notumment : Beil-Jbhrin dans 1..-H. Vixcest, line villa gréco-romaine à Beil-.J̈brin, dans Revue

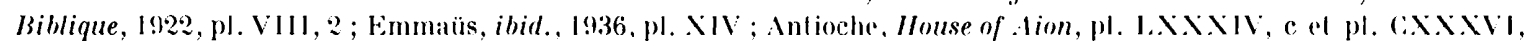
c ; Misis, dans Buml: pl. 65, wc.

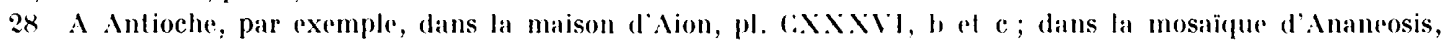
pl. I.XXXI11, a ; dans les Bains F, piece 10, pl. (:IX, b, C.X, d, etc.

29 S. Gramaix, Les mosä̈ques de Timgad, Paris, 1969, n* 74, pl. XX. du ve siecle; n" $184 ;$ n$^{\circ} 194$, pl. I.XVI, no 222.

30 H. Strkx, Recueil I, 1, no $77 \mathrm{~B}$.

31 J. Formati, Les fouilles de Montcarel, dans Comgris archobogique de France, Bordeaux-Bayonne, 1939, p. 190 .

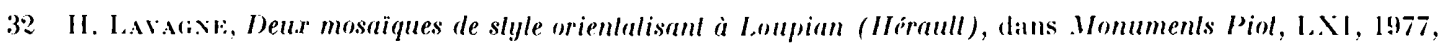
p. $61-86$. 
Trois-Châteaux, la présence de ce décor très luxueux, qui implique une pièce importante, est plus difficile à expliquer. Il peut, certes, s'agir d'une grande demeure édifiée par un citoyen aisé. Pourtant, à pareille époque, l'hypothèse nous paraît peu probable. P.-M. Duval a souligné ${ }^{33}$ l'exiguïté surprenante de la petite forteresse qu'est devenue, le plus souvent, la cité du Bas-Empire. On voit mal dans cette époque de crise, un riche particulier, ou même un haut fonctionnaire, faire bâtir son habitation avec un tel luxe sur les ruines d'un édifice public du Haut-Empire ${ }^{34}$, à un moment où la population de la ville se resserre dans ses murailles ${ }^{35}$. Aussi, sommes-nous amenés à envisager l'hypothèse d'un édifice chrétien qui pourrait correspondre aux débuts de l'évangélisation en pays tricastin. Outre les vraisemblances du contexte historique, quelques arguments peuvent ètre invoqués pour appuyer cette hypothèse.

Tout d'abord, on sait que le quartier Saint-Jean doit son nom à une église de ce titre qui occupe précisément l'emplacement de la maison Vallos où furent mises au jour les mosaïques qui nous occupent. A l'heure actuelle, les seuls restes visibles en élévation sont le cintre d'un arc roman et un chevet plat réemployé dans les maisons modernes. Ils correspondent à l'église donnée aux Templiers en $1136^{36}$, ruinée en 1208 lors de la prise de Saint-Paul par les Albigeois, et probablement modifiée à cette époque par la suppression de l'abside. Mais il est possible de remonter plus haut en rappelant que l'église Saint-Jean existe déjà en 960 , date à laquelle elle est donnée à l'abbaye de Montmajour ${ }^{37}$. Antérieurement à cette date, et du point de vue des textes, nous n'avons que la tradition locale rapportée par N. Delacroix ${ }^{38}$ et A. Sagnier ${ }^{39}$, fixant la construction de la première église de Saint-Paul-Trois-Châteaux à cet emplacement au vi ${ }^{\mathrm{e}}$ siècle. C'est à ce lieu de culte primitif que nous rapporterions les mosaïques, mais en le plaçant plutôt au ve siècle étant donné leur style. Divers indices peuvent peut-être confirmer son existence. On sait que la cathédrale actuelle ne date que des années 1120 pour le début de sa construction ${ }^{40}$. Or, les premiers temps de l'église tricastine remontent aux dernières décennies du Iv $^{\mathrm{e}}$ siècle. Les travaux de J.-R. Palanque ${ }^{41}$, revenant sur l'hypercritique de G. de Manteyer ${ }^{42}$ ont

33 P.-M. Ucval, dans Colloque La Gallia Romana (cf. note 2, supra ', p. 190.

34 Rappelons que c'est à l'époque flavienne que Saint-Paul a reçu le titre de colonie (cf. H. Rortaxi), dans Comples rendus Acad. Inscriptions et Belles-Lettres, 1961, p. 359-363.

35 Il est frappant, à cet égard, de voir que la plus belle mosaique de Saint-Paul, celle d'Hercule délivrant Hésioné, a été trouvée dans une des habitations suburbaines. Ce pavement (Inv. Mos., no 143), d'époque sévérienne, correspond à un agrandissement de la cité au début du ı ${ }^{\mathbf{c}}$ siècle, suivi par la "rétraction " à l'intérieur du rempart au Bas-Empire (cf. Recueil des mosaïques de la Gaule, III, Narbonnaise, 1, Introduction:.

36 J. de Font-RÉaulx, Cartulaire de l'évêché de Saint-Paul-Trois-Châteaux, Valence, 1950, V, p. I68; RıPertMontclar, Carlulaire de la Commanderie de Richerenches de l'ordre du Temple, Avignon, 1907, chartes nos 122, 128, et introduction, p. xcıir.

37 L. Fillet, Les colonies dauphinoises de l'abbaye de Monimajour, Valence, 1891, p. 20, no III; L. BoISSE, Églises el chapelles du Tricastin ou de l'archidiaconé de Saint-Paul-Trois-Châteaux, Pierrelatte, 1964, p. 7.

38 N. Delacroix, op. cil., p. 309.

39 A. SAGNier, op. cit., p. 260, 270, 274.

40 J.-M. Rol quette, Provence romane, La Provence rhodanienne, 1974, p. 73.

41 J.-R. Palaveve, Les évèchés provençaux à l'époque romaine, dans Provence historique, 1950-1951, p. 113; J. Holx, l.es évéchés provençaux de la fin de l'époque romaine à l'avènement des Carolingiens (470-751:, ibid., 1971, p. 415 .

42 G. de Maxteykr, Les origines chrétiennes de la I/e Narbonnaise. Aix-en-Provence, 1925, p. 161. 
établi que les noms de Paul, fondateur de l'évêché des T'ricastini et d'Eusèbe, qui apparaissent en 374 et 394 aux Conciles de Valence et de Nìmes, pouvaient être considérés comme sûrs et non légendaires. On doit donc supposer la présence d'un autre sanctuaire ayant précédé la cathédrale du $\mathrm{xII}^{\mathrm{e}}$ siècle. La situation de l'église Saint-Jean, un peu en retrait dans l'angle sud-ouest du rempart ${ }^{43}$ ne serait pas sans exemples analogues au Bas-Empire. En outre, quelques découvertes archéologiques récentes confirment l'ancienneté de l'occupation religieuse dans toute la zone de la maison Vallos. En 1974-1975, des sondages pratiqués à l'occasion de travaux d'édilité par la Société d'archéologie de Saint-PaulTrois-Châteaux ${ }^{44}$ mirent au jour les restes du bâtiment primitif de Saint-Jean, légèrement décalés au midi du monument roman, ce qui place la cave où furent trouvées les mosaïques en 1889 dans le prolongement de l'axe de celui-ci. En outre, les traces d'un charnier humain accolé à la nef de l'église Saint-Jean furent repérées, ainsi qu'un sarcophage placé sur d'autres sépultures plus profondes que les circonstances ne permirent pas de fouiller ${ }^{45}$. Contre le mur nord de la maison contiguë à l'immeuble Vallos, fut découverte également une croix en pierre de style paléochrétien ${ }^{46}$. Enfin il faut rappeler une constation capitale faite par l'archéologue Bruguier-Roure, en 1885, cinq ans avant l'extraction des mosaïques. Examinant les pavements encore en place, il notait qu'ils "rappelaient les fondements du sanctuaire d'une église ". Cette remarque essentielle, rapportée par A. Sagnier, puis par L. Fillet ${ }^{47}$, n'avait pas été mise en relation avec les restes du sanctuaire primitif découvert par les fouilleurs de la Société d'archéologie de Saint-Paul-Trois-Châteaux qui ignoraient les mosaïques et leur origine réelle. On doit penser que ce n'est pas le décor des pavements, lequel n'a rien de spécifiquement chrétien (même s'il est employé dans les trois ou quatre exemples cités ci-dessus en milieu chrétien), mais bien son organisation qui évoquait pour Bruguier-Roure "les fondements du sanctuaire d'une église». A titre hypothétique, on pourrait envisager, par exemple, que le tapis d'écailles si fréquemment employé par les mosaïstes pour décorer les surfaces semi-circulaires ait pu orner une abside dans une église de taille réduite ${ }^{48}$. Les autres tapis dont nous avions montré l'impossible reconstitution dans un schéma unitaire, pourraient avoir servi à la nef et à des bas-côtés.

P.-A. Février ${ }^{49}$ a insisté avec force sur les dangers d'une interprétation exclusivement

43 Cf. A. Blanchet, Les enceintes romaines de la Gaule, Paris, 1907, p. 277. - A. Grenıer, Manuel d'archéologie gallo-romaine, 1, p. 370 . La situation de l'église primitive près d'une des portes de la ville, et légèrement en retrait. par rapport au rempart a été souvent relevée, notamment par J. Hu вsRT, dans Sellimane di Sludio del Centro italiano di Studi sull'Allo Medioevo, VI, Spolète, 1958, p. 538, 544.

44 Cf. Cl. Borsse, op. cit., p. 34.

45 Ibid., le sarcophage fut pillé la nuit même du dégagement. Restes au Musée Saint-Paul-Trois-Châteaux, calalogue, $\mathrm{n}^{\circ} 56$.

46 Cette croix, dite "Croix Bladignères " du nom du propriétaire acluel, est taillée dans une stèle où l'on voit d'un còté, une figurine du type égyptisant (personnage debout vêtu d'une jupe courte, portant un symbole solaire au-dessus du sein), les bras êtendus. Le personnage fut détouré pour obtenir la forme d'une croix. Au revers, une silhouette malhabilement gravée à l'effigie du Christ imberbe. Collection privée.

47 A. Sagnier, op. cit., p. 273, note 1 - L. Fillet, op. cit., p. 460.

48 Le dernier exemple en date nous parait être le pavement du baptistère (ve siècle ? découvert en 1976 dans les fouilles de Montréal-Séviac (Gers). Inédit.

49 P. A. FÉvrier, Permanence et hérilage de l'antiquité dans la topographie des villes de l'Occident durant le Haut Moyen-Agge, dans Seltimane di Sludio del Centro ltaliano di Sludi sull allo Medioevo, XXI, Spolète, 1974, p. 93-96, $115-117$, p. 271. 
chrétienne des décors de cette époque, en montrant notamment que les salles à abside étaient un phénomène courant au $\mathrm{w}^{\mathrm{e}}$ et au ve siècles dans l'architecture profane ${ }^{50}$ et que les prétendus édifices cultuels, ou même les simples "oratoires". pouraient être sculement "les pièces de réception de maisons appartenant aux élites de la cité ». La mise en garde a toute sa valeur ici et il faudrait de nouvelles fouilles dans les fondations de l'immeuble Vallos pour trouver d'autres preuves archéologiques qui viennent étayer notre argumentation. Néanmoins, l'hypothèse de pavements de la première église Saint-PaulTrois-Châteaux établie au début du ve siècle, dans l'angle du rempart et sur les ruines d'un bâtiment public du Haut-Empire, mérite d'être envisagée. On aurait là un nouvel exemple de la séquence maintes fois attestée où l'on voit se succéder un édifice païen (peut-être religieux si l'on accepte l'hypothèse Sagnier qui y royait le temple de Rome et d'Auguste) et une église paléochrétienne remplacée par une église romane. Quelle que soit la valeur accordée aux présomptions que nous avons proposées, la présence d'un atelier de mosaïques actif vers les années 400 est indéniable. Nous en avons une seconde preuve dans la mosaïque de la chapelle Saint-Jean d'Olonne, entre Séguret et Vaison-laRomaine.

La chapelle Saint-Jean est un petit sanctuaire situé à còté du château d'Olonne ${ }^{51}$ sur un tertre qui domine la vallée de l'Ouvèze et commande la route de Vaison et de Roaix. L'emplacement correspond à peu près au centre de Pagus Deobensis ${ }^{52}$ dont la richesse en témoignages archéologiques est attestée par de nombreuses trouvailles faites au cours du XIX ${ }^{\mathrm{e}}$ siècle.

La mosaïque était déjà connue de l'abbé d'Expilly en 1778 , mais n'a été publiée qu'en $1976^{53}$. Expilly ${ }^{54}$ note qu'à côté des ruines du château "on voit dans la chapelle un reste de mosaïque des temps reculés et c'est un morceau vraiment digne de l'attention des curieux ». Il ajoute ${ }^{55}$ que la chapelle faisait partie d'un ancien monastère des Bénédictins

50 Nous en avons nous-mêmes de bons exemples à loupian. H. Lavagie, R. Prinhomma, I). Rougletre;, La villa gallo-romaine des Prés-Bas à Loupian (Iléraull), dans Gallia, 34, 1976, p. 217-235.

51 Nous tenons à exprimer notre gratitude au propriétaire actuel, $\mathbf{M}$. Derbier, qui nous a reçu avec la plus grande cordialité. -- les Tilla d'Olonne (marquisat érigé en 1755 sont originaires d'Italic (renseignement communiqué par M. J. Barruol ! C'est au début du xixe siècle qu'ils vinrent s’installer en Provence. Ci. également J. Baussax, dans le Bassin du Rhône, 1911-1912, p. 87, qui, d’ailleurs, donne le vocable erroné de Saint-Estive à la chapelle.

52 G. Rarruor., I,es peuples préromains du sud-est de la Gaule, étude de géographie hislorique, Suppl. I, Revue archéologique de Narbonnaise, 1969, p. 283.

53 Dans un article de grande qualité, qui fait partie d'un volume d'Hommages malheureusement peu accessible, J. W. HEM ElriJk et G. J. M. J. TF Riwl.F, Nole on a mosaic in Vaison-la-Romaine, dans Archeologie en Histoirie Opgedrage aan H. Brunsting, Bussum, 1973, p. 327-342. Nous avons eu communication de cel article grâce à l'obligeance de .1. H. Stern.

54 J. J. Expltuy, Dictionnaire géographique, historique el politique des Gaules et de la France, Paris, V, 1778, p. 299 , s.v. Olonne.

55 J. J. Expll.,Y, Histoire ecclésiastique el civile du Comté-Venaissin el de la ville d Avignon, manuscrit inédil conservé au Musée (alvel d'Avignon, n 2462, fol. 443. Mention reprise dans le manuscrit intilulé Iélices d'. lvignon el du Comtat Venaissin, sans nom d'auteur, au Musée Calvet (ms. 2470, fol. 218. (if. également les notes de L. GAp sur l'histoire de Séguret, conservées aux Archives Départementales (Fonds G.\P, F. IV, 2, XII, p. 23. 
qui aurait été cédé aux Templiers de la Commanderie de Roaix ${ }^{56}$. En 1821, les restes du couvent des Templiers étaient encore visibles ${ }^{57}$, et, dès 1857, Courtet ${ }^{58}$ en mentionnant des "aqueducs " et les fragments de mosaïques, proposait d'y voir le siège d'une villa galloromaine en terrasses, agrémentée de bassins. La disposition des lieux aujourd'hui, les canalisations souterraines voûtées en pierre, qui amènent l'eau de la colline, et surtout les divers restes antiques trouvés à proximité inclinent à accepter cette hypothèse ${ }^{59}$.

La mosaïque elle-même, très mutilée (fig. 10) occupe exclusivement la nef du bâtiment ; l'abside et l'aile nord, très remaniées vers 1936 , en sont totalement dépourvues. La pavement est réduit à une frange conservée sur le côté sud adossé à un repli du terrain qui forme une terrasse surplombant la chapelle. Le long de ce mur sud court une bordure en natte (largeur $36 \mathrm{~cm}$ ), à 8 brins polychromes, qui se rétrécit à partir de l'angle sud-est pour devenir tresse à quatre brins sur le côté ouest (fig. 11). Les tonalités sont variées : successivement blanc/rose, rouge/noir, puis blanc/jaune/marron clair/noir ou blanc/beige/vert/noir. La bordure est séparée par une bande blanche $(7 \mathrm{~cm})$ du tapis proprement dit. Celui-ci est composé d'un carré central entouré sur chacun de ses côtés par un groupe de deux carrés (côté $46 \mathrm{~cm}$ ) placés en angles, séparés par un rectangle. Dans les deux carrés, en partie intacts, on retrouve sur fond bleu le quadrilobe de peltes déjà analysé à Saint-Paul-TroisChâteaux, présentant le même motif noir en accent circonflexe et entourant un nœud en sparterie double où alternent les brins de couleurs rouge/jaune/blanc et bleu/jaune/ blanc. La seule différence est que le sommet de la courbure des peltes est surmonté d'un pompon blanc, tandis que les triangles en écoinçons bleus portent un triangle à degrés blanc. Le fragment du rectangle intermédiaire subsistant $(1,13 \mathrm{~m} \times 0,60 \mathrm{~m})$ est à fond polychrome en arc-en-ciel constitué par des lignes de cubes posés en diagonale et reprenant les mêmes couleurs que les brins de la natte. la motif si particulier du quadrilobe de pelte's, la polychromie, l'utilisation de la bordure en natte, le traitement des fonds en arc-enciel reprennent si exactement ce que nous avons vu à Saint-Paul-Trois-Châteaux que l'attribution au même atelier est hors de doute. Si l'on reporte les dimensions des motifs subsistant sur la surface totale de la nef, on obtient la restitution présentée (fig. 12). Il est vrai que la bordure de côté nord serait, dans ce cas, sérieusement réduite, mais les remaniements de l'architecture du bâtiment sur ce point sont tels qu'il est difficile de préciser la largeur de la hordure originelle. C'est d'ailleurs le propre de ces nattes que de pouvoir être utilisées par le mosaïste en fonction de la place qui reste à garnir. La composition du champ carré entre des bordures où alternent carrés et rectangles est extrêmement

56 Aucune précision sur l'otat des batiments dans les archives de l'ordre. CI. J. A. Btrusec, Les Templiers en Provence, dans Provence historique, 9, 1959, p. 17-18.

57 J.-F. Bracher, Voyage d'un anglais dans le dípartement de Vaucluse, Avignon, 1 1 21 , p. 3. Le hénilier était encore en place à celte époque.

58 J. Counter, Diclionnaire des Communes du Vaucluse, Avignon, 1857, p. 309.

59 J. W. Hemelrijk, releve notamment des lampes (type Loeschke I et 11 , de la sigillée signée Verecundus, une sculpture bacchique, et non ioin du château l'inscription CII XII, 1.162 M. Val. Pactoxus. Expilly voulait y voir (op. cit., fol. 443: la "maison de campagne " de M. Valerius Paulinus, procurateur de Narbomaise sous Vespasien (II.-(i. PFlaum, Carrieres procuratoriennes équestres sous le Hanl Empire, Paris 1963, 1, no 40, p. 94-95. Pour Courtet, ce serail la villa de Martius Valerianus, père de sainte Rusticule (556-630 . La nouvelle lecture proposée par IIemelrijk et Riele (op. cit., p. 341: ramenerait plus prosaïquement à un iugarius, marchand de jougs. 


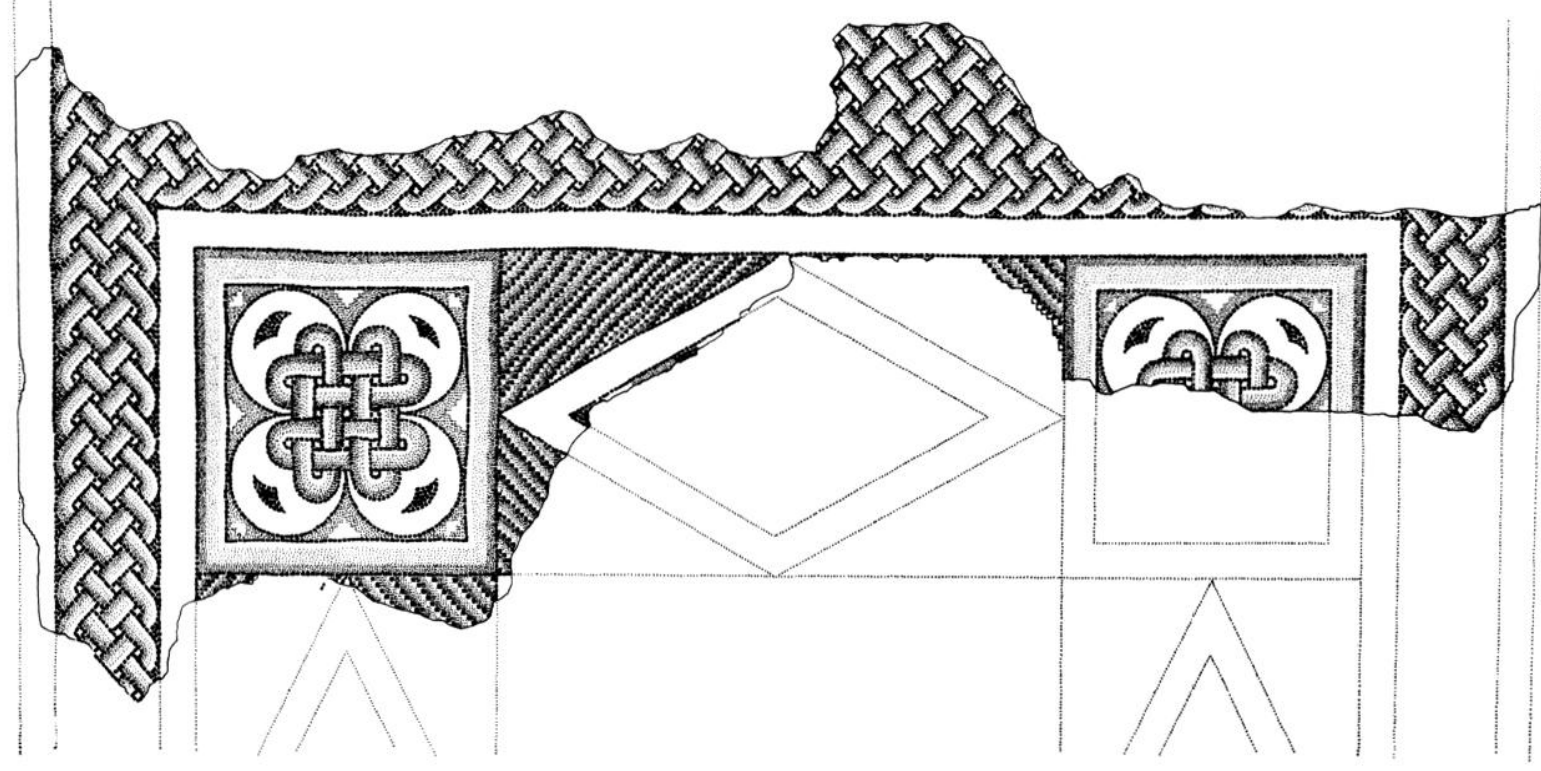

10 Mosaïque de la chapelle saint-Jean a'olonne releve R. Prudhomme .

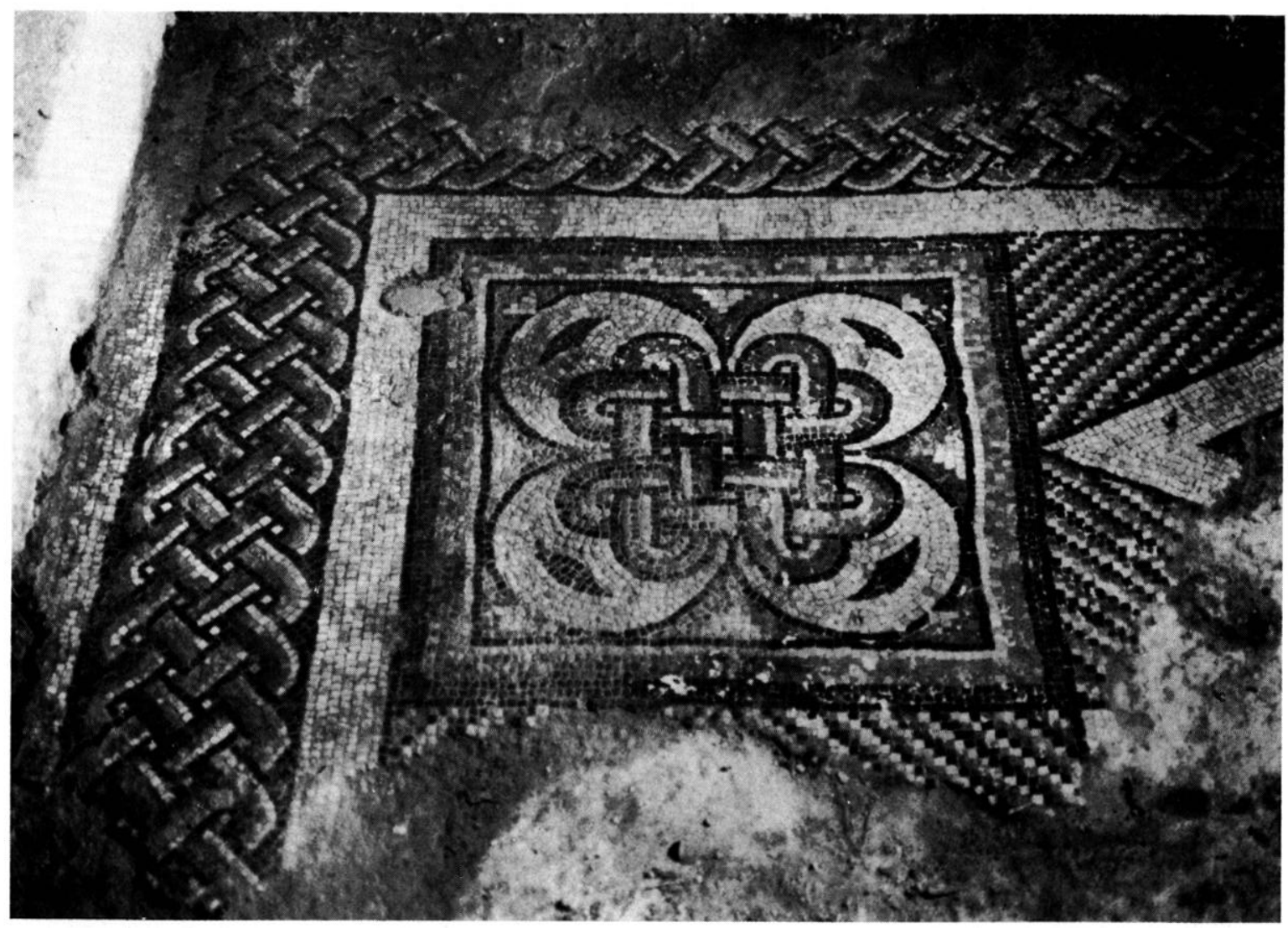

11 Saint-Jean dolonne. Fragment de la mosaique (in silu). 


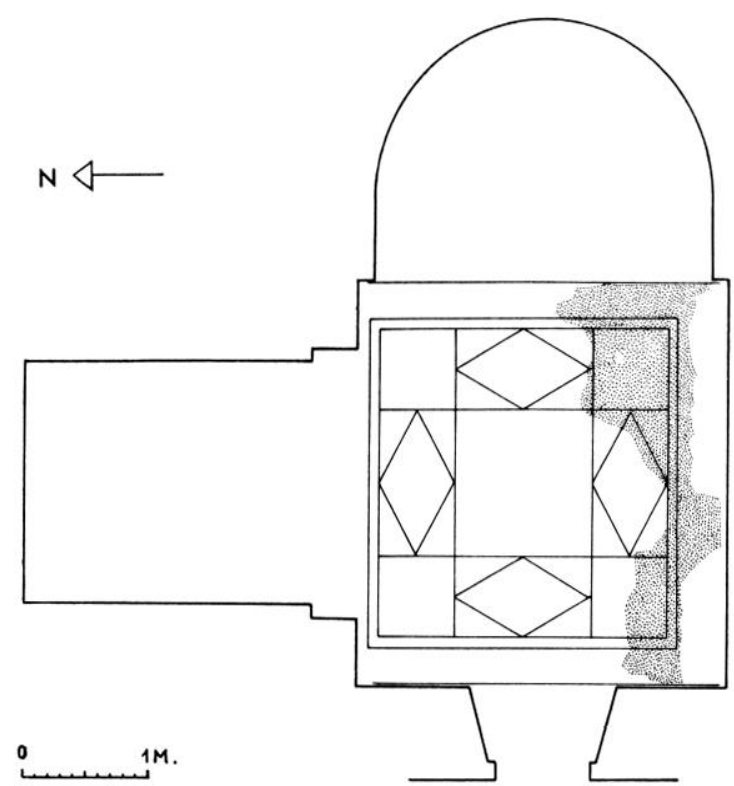

12 Restitution de la mosaique (R. Prudhomme).

courante, notamment à Antioche, par exemple, dans la maison des Bateaux de Psyché et dans celle du terrain Rassim Bey Adali60, ou à Apamée (en 391) ${ }^{61}$, etc. La date que nous proposons (fin $\mathbf{v}^{\mathrm{e}}$-début $\mathrm{v}^{\mathrm{e}}$ siècle), pose les mêmes problèmes d'attribution que dans le cas de Saint-Paul-Trois-Châteaux. Mais ici, nous n'avons aucun témoignage historique ou archéologique qui puisse nous incliner à une interprétation chrétienne. Il peut s'agir du pavement d'un oratoire construit à côté de la villa gallo-romaine et que la chapelle des Bénédictins, puis des Templiers aurait respecté et réutilisé. On expliquerait mieux de cette façon le curieux plan du bàtiment actuel et son orientation.

Les deux séries de mosaïques que nous avons présentées occupent, par leur date, une place importante dans la production pavimentaire de la Narbonnaise. Toutes celles de même époque, connues jusqu'ici, appartiennent à des édifices chrétiens : le petit fragment $\mathrm{du}$ baptistère d'Aix-en-Provence ${ }^{62}$ que nous mettrions au ve siècle, celui qui décore l'intrados de l'atrium de Saint-Victor ${ }^{63}$, ceux (connus par des dessins) du baptistère SaintJean de la Major ${ }^{64}$ à Marseille, et les éléments conservés de la chapelle Saint-Blaise ${ }^{65}$. Nous y ajouterions volontiers les mosaïques de Saint-Paul-Trois-Châteaux, sinon ceux de Saint. Jean d'Olonne. A une époque de crise, comme on l'a écrit ${ }^{66}$ " devant le chaos engendré

60) 1). I.kv, op. cit., pl. (.III f et LXXXIV, a et b. On retrouve cette organisation du champ aussi bien dans la Maison des Mystieres d'Isis (pl. CII, c, d, e que dans celle dite du Buffet Supper (pl. XI.VIII d).

61 V. Vernoorik, Apamea in Syrie, Bruxelles, 1970, fig. 12, 15.

62 F. Briolt, dans Giallia, XVI, 1958, p. 416 , fig. 7.

63 J.-.1. Rotelettr, op. cil., p. 451 .

6.1 11. Strax, Mosaïques de pavement préromanes el romanes en France, dans Cahiers de civilisalion médiétale, 1. 196:, p. 14-15, pl. I, fig. 1 el 2.

65 Ibid., p. 16, pl I, fig. 4.

66 IR. Boykr, l.es habilals de plaine en Provence à la fin de ranliquilé, dans . Icles du 90e Congrès nalional des Sociétés savantes, Nice, 196.j, Paris, 1966, p. 210. 
par les invasions et les troubles de l'Empire d'Occident ", "... le christianisme apparait comme l'ultime recours ". La commande d'un pavement en mosaïque peut être un acte pieux, mais aussi une forme d'évergétisme héritée de traditions antiques ${ }^{67}$. C'est en tout cas le témoignage d'une activité d'ateliers de mosaïstes dans la Provence du début du ve siècle.

Henti lavagne.

67 P.-А. Fivrite, op. cil., p. 44-135. 